\section{Disease-related Risk of Vertebral Fracture During Glucocorticoid Treatment of Collagen Vascular Diseases}

\section{To the Editor:}

Collagen vascular diseases, including systemic lupus erythematosus (SLE), rheumatoid arthritis (RA), Sjögren's syndrome (SS), systemic sclerosis (SSc)/mixed connective tissue disease (MCTD), polymyositis/dermatomyositis (PM/DM), and microscopic polyangiitis, are chronic and sometimes involve multiple organs, and are thus occasionally life-threatening. Immunosuppressive treatment with longterm high-dose glucocorticoids is necessary, but the treatment causes a high incidence of osteoporosis $1,2,3$. Recently, we demonstrated that patients treated with longterm high-dose glucocorticoids have a high risk of symptomatic vertebral fractures in collagen vascular diseases, based on the analysis of the Chiba-Shimoshizu Rheumatic Cohort ${ }^{4,5}$

The chronic and systemic inflammation in patients with RA and SLE has been recognized as causing secondary osteoporosis independently of glucocorticoids ${ }^{6}$, and many studies demonstrated that patients with RA have lower bone mineral density (BMD) and an increased risk of fracture ${ }^{7,8,9}$. However, it was not known whether there is a disease-specific or disease-related risk of osteoporotic fracture under glucocorticoid treatment among the collagen vascular diseases. We investigated the disease-specific or disease-related risk for osteoporotic symptomatic vertebral fracture by focusing on women treated with high-dose glucocorticoids for the long term for collagen vascular diseases, subanalyzing a cohort at Shimoshizu National Hospital in Japan.

The study subjects were newly treated with an initial dose of $>20$ $\mathrm{mg}$ /day prednisolone (PSL) equivalent for at least 6 months without prior prophylactic treatment with bisphosphonate, hormone replacement therapy, or selective estrogen receptor modulator to prevent bone loss. We defined glucocorticoid dose increase as the reintroduction of $\geq 20 \mathrm{mg} /$ day of glucocorticoid (PSL equivalent) due to increased disease activity in patients whose doses were once tapered to $<20 \mathrm{mg}$ /day. Symptomatic vertebral fracture was defined as vertebral deformity that was confirmed by thoracolumbar radiograph when the patient presented with a new backache ${ }^{4,5}$

In our study, we followed 539 women patients on high-dose glucocorticoid therapy (mean initial age $44.6 \pm 16.0 \mathrm{yrs}$ ) and 161 women as nonglucocorticoid controls (mean initial age $49.0 \pm 12.5 \mathrm{yrs}$ ) between 1986 and 2006. The underlying diseases were diverse among the patients in both groups, consisting of SLE (29.9\%), RA (22.4\%), SS (14.6\%), SSc/MCTD $(10.1 \%), \mathrm{PM} / \mathrm{DM}(8.1 \%)$, microscopic polyangiitis $(3.1 \%)$, and others $(11.7 \%)$. In the high-dose glucocorticoid group, the mean initial glucocorticoid dose was $40.2 \mathrm{mg}$ /day and the mean glucocorticoid dose was $14.6 \mathrm{mg} /$ day (both in PSL equivalents). Cumulative glucocorticoid dose was estimated to be $33.6 \mathrm{~g}$ (PSL equivalent). Glucocorticoid pulse therapy was given to $14.7 \%$ of the patients over the entire study. The disease activity-related glucocorticoid dose increase involving $20 \mathrm{mg}$ /day or higher (PSL equivalent) occurred in $40.6 \%$ of the patients. Symptomatic vertebral fractures occurred in $24.7 \%$ of the high-dose glucocorticoid group, significantly higher than the $3.1 \%$ of the no-glucocorticoid group. A Cox model demonstrated that the adjusted hazard ratio (HR) for age, body mass index (BMI), smoking, and alcohol use was 10.64 (95\% CI 4.35-36.03).

We focused on the high-dose glucocorticoid group to analyze the risks for symptomatic vertebral fractures including specific type of disease in glucocorticoid-induced osteoporosis. The fracture group had significantly higher mean age, lower rate of menstruation, shorter followup time, higher mean daily dose, lower cumulative dose, and higher prevalence of glucocorticoid dose increase (Table 1). No significant differences were observed between the 2 groups in BMI, use of alcohol, smoking, initial glucocorticoid dose, or glucocorticoid pulse therapy. We compared the prevalence of each disease between the fracture and no-fracture groups (Table 1); a lower rate of SLE and a higher rate of microscopic polyangiitis were observed in comparison to the no-fracture group. There were no significant
Table 1. Characteristics of study patients at baseline and during glucocorticoid (GC) treatment. Data are expressed as mean \pm SD unless otherwise indicated.

\begin{tabular}{lccc}
\hline Characteristics & $\begin{array}{c}\text { Fracture } \\
\text { Group }\end{array}$ & $\begin{array}{c}\text { No-Fracture } \\
\text { Group }\end{array}$ & $\mathrm{p}$ \\
\hline No. patients & 133 & 406 & \\
Age, yrs & $55.4 \pm 14.1$ & $41.1 \pm 15.0$ & $<0.001^{*}$ \\
Body mass index & $21.3 \pm 3.2$ & $21.1 \pm 3.1$ & $\mathrm{NS} *$ \\
Smoking, \% & 18.0 & 18.1 & $\mathrm{NS} * *$ \\
Alcohol use, \% & 8.6 & 11.3 & $\mathrm{NS} * *$ \\
Menstruation, \% & 30.1 & 67.0 & $<0.001^{* *}$ \\
Followup time, mo & $63.1 \pm 65.3$ & $109.3 \pm 74.4$ & $<0.001^{*}$ \\
Initial dose of PSL, mg/day & $38.3 \pm 15.2$ & $40.8 \pm 14.1$ & $\mathrm{NS}$ \\
Mean dose of PSL, mg/day & $20.1 \pm 11.6$ & $12.8 \pm 6.3$ & $<0.001^{*}$ \\
Cumulative dose of PSL, g & $24.8 \pm 21.8$ & $36.5 \pm 27.2$ & $<0.001^{*}$ \\
GC pulse therapy, \% & 15.1 & 13.5 & $\mathrm{NS} *$ \\
No. GC dose increases & & & \\
$\quad$ 0/1/2/3/4 & $58 / 51 / 16 / 6 / 2$ & $262 / 81 / 39 / 20 / 4$ & $<0.001^{* *}$ \\
SLE, \% & 27.8 & 42.1 & $<0.01^{* *}$ \\
RA, \% & 9.0 & 6.9 & $\mathrm{NS} * *$ \\
SS, \% & 10.5 & 15.8 & $\mathrm{NS} * *$ \\
SSc/MCTD, \% & 15.8 & 11.1 & $\mathrm{NS} * *$ \\
PM/DM, \% & 12.0 & 10.1 & NS** \\
Microscopic polyangiitis, \% & 9.0 & 2.5 & $<0.01^{* *}$ \\
\hline
\end{tabular}

PSL: prednisolone; SSc: systemic sclerosis; MCTD: mixed connective tissue disease; PM/DM: polymyositis/ dermatomyositis; SLE: systemic lupus erythematosus; RA: rheumatoid arthritis. * Unpaired t test. ** Chi-squared test.

differences between the 2 groups in rates of RA, SS, SSc/MCTD, and $\mathrm{PM} / \mathrm{DM}$ (Table 1).

To analyze the disease-related risk among the collagen vascular diseases, the risk factors were evaluated with Cox regression models (Table 2). The risk for symptomatic vertebral fracture was independently higher with every 10-year increment of initial age, with HR $1.83(95 \%$ CI $1.51-2.23$ ), with every $10 \mathrm{mg} /$ day of initial glucocorticoid dose (PSL equivalent; HR 1.44, 95\% CI 1.22-1.69), with every glucocorticoid-dose increase (HR 2.52, 95\% CI 2.04-3.12), in SLE (HR 2.02, 95\% CI 1.08-3.76), in SSc/MCTD (HR 2.47, 95\% CI 1.27-4.80), and in microscopic polyangiitis (HR 2.87, 95\% CI 1.30-6.32). However, symptomatic vertebral fracture risk was independently higher with a decrease of each gram of cumulative glucocorticoid dose (PSL equivalent; HR 0.92, 95\% CI $0.91-0.94)$.

Chronic and systemic inflammation has been known to cause secondary osteoporosis independently of glucocorticoid in systemic autoimmune diseases such as RA and $\mathrm{SLE}^{6}$. In addition, a study demonstrated that patients with SSc may have increased risk of osteoporosis ${ }^{10}$, although it has also been reported that there is no strong evidence in the literature for consistently lower BMD scores in $\mathrm{SSc}^{11}$. To analyze disease-related fracture risks, we focused on the 6 most frequent $(>2.5 \%)$ collagen vascular diseases found in our study: SLE, RA, SS, SSc/MCTD, PM/DM, and microscopic polyangiitis.

Although the rate of SLE in the patients with vertebral fracture was significantly lower than in those without SLE, a Cox regression model conversely demonstrated that SLE is an independent risk factor in our model. This discrepancy might be attributed to age difference, as the patients with SLE were significantly younger $(36.1 \pm 13.5 \mathrm{yrs})$ than those without it $(50.0 \pm 15.2 \mathrm{yrs} ; \mathrm{p}<0.001)$. Among the collagen vascular diseases analyzed, SLE, MCTD/SSc, and microscopic polyangiitis were independent risk factors as confirmed by Cox regression analysis.

There are many risk factors for fracture and bone loss in collagen vas-

Personal non-commercial use only. The Journal of Rheumatology Copyright $@$ $~ 2011$. All rights reserved 
Table 2. Evaluation of risk for symptomatic vertebral fracture using Cox proportional hazard regression (HR) model.

\begin{tabular}{lcc}
\hline Variables & HR $(95 \%$ CI $)$ & $\mathrm{p}$ \\
\hline Initial age (every 10 yrs) & $1.83(1.51-2.23)$ & $<0.0001$ \\
BMI (every 1 BMI) & $0.99(0.93-1.05)$ & 0.686 \\
Smoking & $1.18(0.71-1.98)$ & 0.525 \\
Alcohol use & $1.40(0.730-2.67)$ & 0.315 \\
Menstruation & $0.93(0.51-1.68)$ & 0.800 \\
Initial dose of PSL (every 10 mg/day) & $1.44(1.22-1.69)$ & $<0.001$ \\
GC pulse therapy & $1.36(0.79-2.35)$ & 0.265 \\
No. GC dose increases & $2.52(2.04-3.12)$ & $<0.001$ \\
Cumulative dose of PSL (every 1 g) & $0.92(0.91-0.94)$ & $<0.001$ \\
SLE & $2.02(1.08-3.76)$ & $<0.05$ \\
RA & $1.67(0.82-3.38)$ & 0.158 \\
SS & $1.15(0.55-2.38)$ & 0.715 \\
SSc/MCTD & $2.47(1.27-4.80)$ & $<0.01$ \\
PM/DM & $1.28(0.63-2.60)$ & 0.496 \\
Microscopic polyangiitis & $2.87(1.30-6.32)$ & $<0.01$ \\
\hline
\end{tabular}

BMI: body mass index; PSL: prednisolone; GC: glucocorticoid; SLE: systemic lupus erythematosus; RA: rheumatoid arthritis; SS: Sjögren's syndrome; SSc: systemic sclerosis; MCTD: mixed connective tissue disease; PM/DM: polymyositis/dermatomyositis.

cular diseases independent of glucocorticoid ${ }^{3,6}$. Some examples are reduced motility, renal impairment, induction of bone-resorbing chemokines and cytokines, endocrine disorders (amenorrhea, premature menopause, low plasma androgen level, and hyperprolactinemia), sunshine avoidance (low vitamin $\mathrm{D}_{3}$ status), chronic anticoagulation, and immunomodulant/suppressive drugs. Studies have attracted attention to interactions between the immune and skeletal systems (osteoimmunology). In these interactions, not only is the release of chemokines and cytokines [receptor activator of nuclear factor- $\kappa \mathrm{B}$ ligand, interleukin 1 (IL-1), IL-6, IL-11, IL-15, IL-17, monocyte colony-stimulating factor, tumor necrosis factor- $\alpha$, prostaglandins, and parathyroid hormone-related peptide] critical, but so also is direct cell-cell contact ${ }^{6,12}$. In any event, our study did not clarify the precise pathophysiological mechanism of symptomatic vertebral fractures during treatment with high-dose glucocorticoid in collagen vascular diseases.

It was of major interest, as well as unexpected, that SLE, SSc/MCTD, and microscopic polyangiitis were found to be independent risk factors of symptomatic vertebral fractures. MCTD is characterized by manifestations that overlap SLE, SSc, inflammatory myopathy, and RA ${ }^{13}$. Apart from RA, it is known that SLE, SSc, and systemic vasculitides including microscopic polyangiitis are associated with vascular inflammation, altered angiogenesis, and increased cardiovascular morbidity and mortality ${ }^{14}$. Therefore, it might be reasonable to speculate that vascular involvement in these systemic autoimmune diseases impaired skeletal functions such as osteoblastic lineage and osteocytes, leading to bone fragility.

In our study, 3 of the independent risk factors related to glucocorticoid were higher initial dose, greater number of glucocorticoid dose increases, and lower cumulative glucocorticoid dose. Positive correlations between cumulative dose and fracture risk were contrarily found in previous studies $^{15,16}$. Although it is difficult to determine the basis for the negative association, the presence of some genetic or environmental risk factor(s) in the symptomatic vertebral fracture group is suggested. These factors may shorten the duration until vertebral fracture and thus reduce the cumulative dose of glucocorticoid. As a result, the cumulative dose might be negatively associated with fracture risk.

Our study has several limitations. We started from 1986 without a baseline radiograph and measurement of $\mathrm{BMD}$, and we used symptomatic vertebral fracture determined by clinical observation followed by radiograph as an endpoint. Although it has been known that prior fracture is one of the important fracture risk factors in glucocorticoid-induced osteoporosis ${ }^{17,18}$, we could not analyze the prior fracture as a glucocorticoid-induced fracture risk. In addition, because vertebral fracture associated with glucocorticoid therapy is often asymptomatic ${ }^{19,20}$, there may have been more fractures than we observed.

Although the correlation between BMD and glucocorticoid-induced fracture risk has been discussed for years, we had no data on BMD. Studies have suggested that bone strength is determined not only by BMD but also by bone quality, and bone quality rather than mineral density may be critical in glucocorticoid-induced fracture risk ${ }^{18}$.

The vertebral fracture risks of SLE, MCTD/SSc, and microscopic polyangiitis were independently higher during the high-dose glucocorticoid treatment.

ICHIRO TATSUNO, MD, PhD; SAWAKO SUZUKI, MD, PhD; TOMOHIKO YOSHIDA, MD, PhD; HIDEKAZU NAGANO, MD; NAOKO HASHIMOTO, MD, PhD; TAKAFUMI MAYAMA, MD, PhD; TOMOAKI TANAKA, MD, PhD, Department of Clinical Cell Biology, Graduate School of Medicine, Chiba University, and the Department of Clinical Endocrinology and Metabolism, Chiba University Hospital; TAKAO SUGIYAMA, MD, Department of Rheumatology, Shimoshizu National Hospital, Chiba, Japan.

Address correspondence to Dr. I. Tatsuno, Department of Clinical Cell Biology, Chiba University Graduate School of Medicine, 1-8-1 Inohana, Chuo-ku, Chiba, 260-8670, Japan.

E-mail: ichiro-tatsuno@faculty.chiba-u.jp

\section{REFERENCES}

1. Jardinet D, Lefebvre C, Depresseux G, Lambert M, Devogelaer JP, Houssiau FA. Longitudinal analysis of bone mineral density in pre-menopausal female systemic lupus erythematosus patients: deleterious role of glucocorticoid therapy at the lumbar spine. Rheumatology 2000;39:389-92.

2. Nagai S, Izumi T. Immunopathology of collagen vascular disease. Curr Opin Pulm Med 1997;3:356-60.

3. Sinigaglia L, Varenna M, Girasole G, Bianchi G. Epidemiology of osteoporosis in rheumatic diseases. Rheum Dis Clin North Am 2006;32:631-58.

4. Sugiyama T, Tatsuno I, Suzuki S, Yoshida T, Tanaka T, Sueishi M, et al. Incidence of symptomatic vertebral fracture with high-dose glucocorticoid treatment in the Chiba-Shimoshizu Rheumatic Cohort between 1986 and 2006. Endocr J 2009;56:591-9.

5. Tatsuno I, Sugiyama T, Suzuki S, Yoshida T, Tanaka T, Sueishi M, et al. Age-dependent early complication of symptomatic vertebral fracture with high-dose glucocorticoid in treatment for collagen vascular diseases. J Clin Endocrinol Metab 2009;94:1671-7.

6. Walsh NC, Crotti TN, Goldring SR, Gravallese EM. Rheumatic diseases: the effects of inflammation on bone. Immunol Rev 2005;208:228-51.

7. Haugeberg G, Orstavik RE, Kvien TK. Effects of rheumatoid arthritis on bone. Curr Opin Rheumatol 2003;15:469-75.

8. Lodder MC, de Jong Z, Kostense PJ, Molenaar ET, Staal K, Voskuyl AE, et al. Bone mineral density in patients with rheumatoid arthritis: relation between disease severity and low bone mineral density. Ann Rheum Dis 2004;63:1576-80.

9. Orstavik RE, Haugeberg G, Uhlig T, Mowinckel P, Falch JA, Halse $\mathrm{JI}$, et al. Incidence of vertebral deformities in 255 female rheumatoid arthritis patients measured by morphometric X-ray absorptiometry. Osteoporos Int 2005; 16:35-42.

10. Yuen SY, Rochwerg B, Ouimet J, Pope JE. Patients with scleroderma may have increased risk of osteoporosis. A comparison to rheumatoid arthritis and noninflammatory musculoskeletal conditions. J Rheumatol 2008;35:1073-8. 
11. Loucks J, Pope JE. Osteoporosis in scleroderma. Semin Arthritis Rheum 2005;34:678-82.

12. Caetano-Lopes J, Canhao H, Fonseca JE. Osteoimmunology - the hidden immune regulation of bone. Autoimmun Rev 2009;8:250-5.

13. Hoffman RW, Maldonado ME. Immune pathogenesis of mixed connective tissue disease: a short analytical review. Clin Immunol 2008;128:8-17.

14. Szekanecz Z, Koch AE. Vascular involvement in rheumatic diseases: 'vascular rheumatology'. Arthritis Res Ther 2008;10:224

15. Dykman TR, Gluck OS, Murphy WA, Hahn TJ, Hahn BH. Evaluation of factors associated with glucocorticoid-induced osteopenia in patients with rheumatic diseases. Arthritis Rheum 1985;28:361-8.

16. McEvoy CE, Ensrud KE, Bender E, Genant HK, Yu W, Griffith $\mathrm{JM}$, et al. Association between corticosteroid use and vertebral fractures in older men with chronic obstructive pulmonary disease. Am J Respir Crit Care Med 1998;3 Pt 1:704-9.
17. Kanis JA, Johansson H, Oden A, Johnell O, de Laet C, Melton III $\mathrm{LJ}$, et al. A meta-analysis of prior corticosteroid use and fracture risk. J Bone Miner Res 2004;19:893-9.

18. Kanis JA, Stevenson M, McCloskey EV, Davis S, Lloyd-Jones M. Glucocorticoid-induced osteoporosis: a systematic review and cost-utility analysis. Health Technol Assess 2007;11:1-231.

19. Angeli A, Guglielmi G, Dovio A, Capelli G, de Feo D, Giannini S, et al. High prevalence of asymptomatic vertebral fractures in post-menopausal women receiving chronic glucocorticoid therapy: a cross-sectional outpatient study. Bone 2006;39:253-9.

20. Canalis E, Mazziotti G, Giustina A, Bilezikian JP.

Glucocorticoid-induced osteoporosis: pathophysiology and therapy. Osteoporos Int 2007;18:1319-28.

J Rheumatol 2011;38:10, doi:10.3899/jrheum.110412 